\title{
Variational approximation and the use of collective coordinates
}

\author{
J. H. P. Dawes ${ }^{1}$ and H. Susanto ${ }^{2}$ \\ ${ }^{1}$ Department of Mathematical Sciences, University of Bath, Claverton Down, Bath BA2 7AY, United Kingdom \\ ${ }^{2}$ School of Mathematical Sciences, University of Nottingham, University Park, Nottingham, NG7 2RD, United Kingdom
}

(Received 26 September 2012; revised manuscript received 2 April 2013; published 4 June 2013)

\begin{abstract}
We consider propagating, spatially localized waves in a class of equations that contain variational and nonvariational terms. The dynamics of the waves is analyzed through a collective coordinate approach. Motivated by the variational approximation, we show that there is a natural choice of projection onto collective variables for reducing the governing (nonlinear) partial differential equation (PDE) to coupled ordinary differential equations (ODEs). This projection produces ODEs whose solutions are exactly the stationary states of the effective Lagrangian that would be considered in applying the variational approximation method. We illustrate our approach by applying it to a modified Fisher equation for a traveling front, containing a non-constant-coefficient nonlinear term. We present numerical results that show that our proposed projection captures both the equilibria and the dynamics of the PDE much more closely than previously proposed projections.
\end{abstract}

DOI: 10.1103/PhysRevE.87.063202

PACS number(s): 05.45.Yv, 46.15.Cc, 02.30.Jr

\section{INTRODUCTION}

The dynamics of localized waves in completely integrable systems can be analyzed notably using the inverse scattering, or inverse spectral, transform. The method was first proposed in [1] to solve the Korteweg-de Vries equation [2,3]. It provides an explicit integral representation of the solution that has then been applied to many nonlinear evolution equations, such as the sine-Gordon equation $[4,5]$ and the nonlinear Schrödinger equation [6,7] [see, e.g., [8] for a list of other "integrable" partial differential equations (PDEs)]. Localized waves of integrable systems are also often referred to as solitons [9]. Initially studied in autonomous systems, the concept of integrability and the notion of a "soliton" have been extended to nonautonomous equations [10-12].

Even though exact integrability plays an important role in the theory of nonlinear waves, most equations modeling physical systems are not integrable. For systems that are "close" to integrable, the inverse scattering transform method can still be employed. By treating the terms that cause nonintegrability as a perturbation, an inverse scattering perturbation theory was introduced in [13-15]. See [16] for a comprehensive review of the method and its applications. The main limitation of the perturbation theory is, naturally, that it relies on the integrability of the unperturbed system.

A different approach is provided by the so-called collective variable or collective coordinate method. By allowing the parameters of the localized wave, such as its position or width, to vary as a function of time, one then reduces the solution dynamics from being governed by a PDE into coupled ordinary differential equations (ODEs). The reduction can be obtained in several different ways, for instance, by exploiting conservation laws of the unperturbed equations or Lagrangian formalisms. Thus, instead of integrability, these direct perturbation methods require conserved quantities. In the first of these methods, also called energetic analysis, the dynamics of the solution's parameters are governed by the rate of change of the integral invariants, which should vanish in the unperturbed case (see, e.g., [16-19]). In the Lagrangian or variational method, the solution's parameters act as new canonical coordinates that have to satisfy the
Euler-Lagrange equations (see, e.g., [20-23]). The estimation of optimal parameters through the construction of variational principles has a long history, scattered across many different application areas, including, for example, fluid mechanics, where Chandrasekhar [24] used a variational approach to estimate the critical Rayleigh number for the onset of thermally driven instability in a layer of viscous fluid. We refer the reader to Ref. [25] for a review of collective coordinate approaches.

Recently, Caputo and Sarels [26] considered the interaction of a reaction-diffusion front and a localized defect. A theoretical analysis based on the collective coordinate approach was carried out. Instead of using the Lagrangian formulation, Caputo and Sarels derived equations of motion for the parameters by using conservation-quantity-like integrals, which were obtained by substituting the ansatz for the solution into the PDE and averaging it after multiplications with a function (see also [27-29] for a similar approach). However, it is important to note that the multiplying functions were, in that study, chosen rather arbitrarily. Here, we show that there is a natural choice of functions for projection onto the collective coordinates, based on the variational formulation. Our proposed method is similar to that of [30-33] when the nonvariational terms are small. It is also similar to the so-called nonlinear Ritz method for dissipative systems [34] (see also the Supplemental Material of [35]), which is a generalization of the Whitham principle for periodic waves [36]. The Ritz principle separates the governing equations into a variational functional that defines a free energy and a functional that depends on the time derivative only. Our proposed method extends the principle to the case when the free energy function may also contain nonvariational terms.

We then apply our proposed method to a modified Fisher equation, i.e., that considered in [26]. The reader is referred to [26] and references therein for the relevance and the physical applications of the equations. We show below that our method, which in the present example coincides with that in [34], provides a very good approximation to the numerical results. Due to the inhomogeneity, the front can be pinned. The pinning estimate will never be excellent because the front departs from the form of our ansatz as it crosses the inhomogeneity. 
Radiation of continuous waves is also disregarded as a typical characteristic of collective variable approaches. The ansatz we use is expected to apply when the inhomogeneity is slowly varying, but not otherwise. Nevertheless, notably we observe that even when the inhomogeneity is a $\delta$ function, we obtain good quantitative agreement between the approximation and numerical solution of the full problem.

The remainder of the paper is structured as follows. In Sec. II we write out the general expressions that indicate how one can extend the variational approximation into a set of dynamical equations for the time evolution of collective coordinates. In Sec. III we apply our method to a specific problem: the propagation of fronts in a spatially inhomogeneous medium. In Sec. IV we show that our results here on the dynamics of the problem are a substantial improvement over previous work, comparing them with numerical simulation and the results of [26]. A summary and concluding remarks are contained in Sec. V.

\section{DYNAMICS AND THE VARIATIONAL APPROXIMATION}

Let $u(x, t)$, for $x \in \Omega \subseteq \mathbb{R}$ and $t \geqslant 0$, satisfy the PDE

$$
u_{t}=f_{V}\left(x, u, u_{x}, u_{x x}\right)+f_{N V}\left(x, u, u_{x}, u_{x x}\right),
$$

which is second order and (in general) inhomogeneous in space. We note in passing that our results extend naturally to PDEs of higher order in space, but (1) is sufficient here to describe our approach. We assume that suitable boundary conditions are specified on $\left.u\right|_{\partial \Omega}$, for example, Neumann boundary conditions, $u_{x}=0$. In writing (1) we have split the right-hand side into variational and nonvariational parts, i.e., there exists a function $F\left(x, u, u_{x}\right)$ such that

$$
f_{V}\left(x, u, u_{x}, u_{x x}\right)=\frac{\partial F}{\partial u}-\frac{d}{d x} \frac{\partial F}{\partial u_{x}} .
$$

In the variational case (that is, assuming that $f_{N V} \equiv 0$ ) we observe that stationary solutions of (1) are extrema of the functional $\mathcal{L}:=\int_{\Omega}-F\left(x, u, u_{x}\right) d x$ taken over a space of suitable functions $\mathcal{D} \ni u$. The variational approximation consists of simplifying the problem of finding stationary solutions of (1) by restricting the space of functions $\mathcal{D}$ to a family of functions $u=U\left(x, A_{1}, \ldots, A_{n}\right)$ described by a finite number of parameters $A_{1}, \ldots, A_{n}$. Such functions span a finite dimensional subspace $\mathcal{D}_{A} \subset \mathcal{D}$. We denote the restricted Lagrangian by $\mathcal{L}_{\text {eff }}:=\left.\mathcal{L}\right|_{\mathcal{D}_{A}}$. Extrema for the effective Lagrangian satisfy the finite number of constraints $\partial \mathcal{L}_{\text {eff }} / \partial A_{i}=0$ for $i=1, \ldots, n$. Explicitly, these constraints can be written as

$$
\frac{\partial \mathcal{L}_{\mathrm{eff}}}{\partial A_{i}}=\int_{\Omega} \frac{\partial U}{\partial A_{i}}\left[\frac{d}{d x} \frac{\partial F}{\partial U_{x}}-\frac{\partial F}{\partial U}\right] d x=0, \quad i=1, \ldots, n .
$$

Suppose now that we allow evolution in time of the parameters $A_{i}(t)$. Restricting (1) to $\mathcal{D}_{A}$ we now have

$$
\frac{\partial F}{\partial U}-\frac{d}{d x} \frac{\partial F}{\partial U_{x}}=\sum_{j=1}^{n} \frac{\partial U}{\partial A_{j}} \dot{A}_{j}-f_{N V}\left(x, U, U_{x}, U_{x x}\right),
$$

where $\dot{A}_{j} \equiv d A_{j} / d t$, so that for each $i$ we have

$$
\begin{aligned}
\frac{\partial \mathcal{L}_{\mathrm{eff}}}{\partial A_{i}} & =\int_{\Omega} \frac{\partial U}{\partial A_{i}}\left[\frac{d}{d x} \frac{\partial F}{\partial U_{x}}-\frac{\partial F}{\partial U}\right] d x, \\
& =\int_{\Omega} \frac{\partial U}{\partial A_{i}}\left[f_{N V}-\sum_{j=1}^{n} \frac{\partial U}{\partial A_{j}} \dot{A}_{j}\right] d x .
\end{aligned}
$$

Interchanging the integration and finite summation we obtain a set of ordinary differential equations for the parameters $A_{j}$ :

$$
\sum_{j=1}^{n} M_{i j} \dot{A}_{j}=-\frac{\partial \mathcal{L}_{\mathrm{eff}}}{\partial A_{i}}+\int_{\Omega} \frac{\partial U}{\partial A_{i}} f_{N V} d x
$$

where the coupling matrix $M_{i j}=\int_{\Omega} \frac{\partial U}{\partial A_{i}} \frac{\partial U}{\partial A_{j}} d x$ is clearly symmetric. We note that the variational setup also shows that when $f_{N V} \equiv 0, \mathcal{L}_{\text {eff }}$ is a nonincreasing function of time, since

$$
\begin{aligned}
\frac{d \mathcal{L}_{\mathrm{eff}}}{d t} & =\sum_{i=1}^{n} \dot{A}_{i} \frac{\partial \mathcal{L}_{\mathrm{eff}}}{\partial A_{i}} \\
& =\int_{\Omega} \sum_{i=1}^{n} \dot{A}_{i} \frac{\partial U}{\partial A_{i}}\left[\frac{d}{d x} \frac{\partial F}{\partial U_{x}}-\frac{\partial F}{\partial U}\right] d x \\
& =-\int_{\Omega}\left(\sum_{i=1}^{n} \dot{A}_{i} \frac{\partial U}{\partial A_{i}}\right)^{2} d x \leqslant 0 .
\end{aligned}
$$

We note that, equivalently, $\frac{d \mathcal{L}_{\text {eff }}}{d t}$ can be expressed in terms of the coupling matrix $M$ :

$$
\frac{d \mathcal{L}_{\mathrm{eff}}}{d t}=-\sum_{i, j=1}^{n} \dot{A}_{i} M_{i j} \dot{A}_{j}
$$

\section{PROPAGATING FRONTS WITH SPATIAL INHOMOGENEITY}

In this section we apply the general formulation from Sec. II to the specific problem of a stable traveling front in a scalar nonlinear diffusion equation with a bistable cubic nonlinearity. We introduce a space-dependent factor multiplying the nonlinear term and investigate the variation in front propagation speed that this causes. Consider the PDE

$$
u_{t}=u_{x x}+s(x) u(1-u)(u-a)=f_{V}\left(x, u, u_{x}, u_{x x}\right),
$$

having constant (and linearly stable) solutions $u(x, t)=1$ and $u(x, t)=0$, and a linearly unstable solution $u(x, t)=a$. We take the parameter $a$ to satisfy $0<a<1 / 2$ without loss of generality (the dynamics for $1 / 2<a<1$ is equivalent after the change of variable $u=1-\tilde{u})$. We remark in passing that even though the PDE (4) is not a constant coefficient equation, it is nevertheless variational since it can be written in the form (1) and (2) with $f_{N V} \equiv 0$ and

$$
F=-\frac{1}{2} u_{x}^{2}-s(x)\left(\frac{1}{4} u^{4}-\frac{1}{3}(1+a) u^{3}+\frac{a}{2} u^{2}\right) .
$$

In the homogeneous case where $s(x) \equiv s_{0}$ is constant there is a stable, and monotonically decreasing, traveling front (or "kink") solution

$$
u(x, t)=\left\{1+\exp \left[\sqrt{s_{0} / 2}(x-c t)\right]\right\}^{-1},
$$


with speed $c=\sqrt{s_{0} / 2}(1-2 a)>0$. We note that a symmetrically related monotonically increasing front also exists.

When we allow $s(x)$ to vary in space, we anticipate that if $s(x)$ varies slowly compared to the characteristic width of the front $\sim \sqrt{2 / s}$ then the solution will remain close to a monotonic front of the form (5), for example being well approximated by an ansatz of the form

$$
u(x, t)=\left\{1+\exp \left[\left(x-x_{0}(t)\right] / w(t)\right)\right\}^{-1} .
$$

The method of Sec. II then enables us to deduce ODEs for the parameters $x_{0}(t)$ and $w(t)$ characterizing the position and width of the traveling front as it passes, for example, over a region in which $s(x)$ varies.

To simplify notation, following Caputo and Sarels [26], we write our ansatz (6) in the form

$$
u(x, t)=U(z)=[1+\exp (z)]^{-1}, \quad z=\left(x-x_{0}\right) / w .
$$

The ODEs (3) for the evolution of the parameters $x_{0}$ and $w$ take the forms

$$
\begin{aligned}
& \dot{x}_{0} \int_{-\infty}^{\infty} \frac{\partial U}{\partial x_{0}} \frac{\partial U}{\partial x_{0}} d x+\dot{w} \int_{-\infty}^{\infty} \frac{\partial U}{\partial x_{0}} \frac{\partial U}{\partial w} d x=\int_{-\infty}^{\infty} \frac{\partial U}{\partial x_{0}} f_{V} d x, \\
& \dot{x}_{0} \int_{-\infty}^{\infty} \frac{\partial U}{\partial w} \frac{\partial U}{\partial x_{0}} d x+\dot{w} \int_{-\infty}^{\infty} \frac{\partial U}{\partial w} \frac{\partial U}{\partial w} d x=\int_{-\infty}^{\infty} \frac{\partial U}{\partial w} f_{V} d x .
\end{aligned}
$$

Compare (7) and (8) with (14) and (15) of [26]. The integrals on the right-hand side evaluate to yield

$$
\begin{aligned}
\int_{-\infty}^{\infty}\left(\frac{\partial U}{\partial x_{0}}\right)^{2} d x & =\frac{1}{w} \int_{-\infty}^{\infty} \frac{e^{2 z}}{\left(1+e^{z}\right)^{4}} d z=\frac{1}{6 w} \\
\int_{-\infty}^{\infty} \frac{\partial U}{\partial x_{0}} \frac{\partial U}{\partial w} d x & =\frac{1}{w} \int_{-\infty}^{\infty} \frac{z e^{2 z}}{\left(1+e^{z}\right)^{4}} d z=0 \\
\int_{-\infty}^{\infty} \frac{\partial U}{\partial w} \frac{\partial U}{\partial w} d x & =\frac{1}{w} \int_{-\infty}^{\infty} \frac{z^{2} e^{2 z}}{\left(1+e^{z}\right)^{4}} d z=\frac{1}{3 w}\left(\frac{\pi^{2}}{6}-1\right) .
\end{aligned}
$$

That (9) vanishes can be seen immediately since the integrand is odd-symmetric. Hence in this case the coupling matrix $M$ happens to be diagonal. Now we turn to the right-hand sides of (7) and (8). The contributions from the diffusive term $u_{x x}$ in $f_{V}$ turn out to be zero [since our ansatz $U(z)$ has zero slope as $x \rightarrow \pm \infty]$ and $1 /\left(12 w^{3}\right)$, respectively. The nonlinear terms in $f_{V}$ make contributions that depend on the (as yet unspecified) form of $s(x)$. We obtain

$$
\begin{gathered}
\frac{\dot{x}_{0}}{6 w}=\int_{-\infty}^{\infty} S \frac{e^{2 z}\left(1-a-a e^{z}\right)}{\left(1+e^{z}\right)^{5}} d z \\
\frac{\dot{w}}{3 w}\left(\frac{\pi^{2}}{6}-1\right)=\frac{1}{12 w^{3}}+\frac{1}{w} \int_{-\infty}^{\infty} S \frac{z e^{2 z}\left(1-a-a e^{z}\right)}{\left(1+e^{z}\right)^{5}} d z
\end{gathered}
$$

where $S=s\left(z w+x_{0}\right)$. We note that this form is similar to that obtained in [26]; the difference is that the authors there obtained a pair of ODEs for $x_{0}(t)$ and $w(t)$ in a more arbitrary fashion by multiplying (4) by 1 and by $u$, respectively, before integrating over $\mathbb{R}$. In this way Caputo and Sarels obtained the ODEs

$$
\begin{gathered}
\dot{x}_{0}=w \int_{-\infty}^{\infty} S \frac{e^{z}\left(1-a-a e^{z}\right)}{\left(1+e^{z}\right)^{3}} d z \\
\dot{w}=\frac{1}{3 w}-w \int_{-\infty}^{\infty} S \frac{e^{z}\left(1-e^{z}\right)\left(1-a-a e^{z}\right)}{\left(1+e^{z}\right)^{4}} d z .
\end{gathered}
$$

These are Eqs. (19) in [26].

\section{NUMERICAL COMPARISONS}

In this section we present a small number of comparisons between the two reduced "collective coordinates" descriptions of the front dynamics: Eqs. (10) and (11) derived here and (12) and (13) derived in [26].

\section{A. Slowly varying heterogeneity $s(x)$}

An obvious consistency check of both pairs of ODEs is that they reduce to the known exact front solution (5) when $s(x)$ is constant. Moreover, this should be the asymptotic behavior of the system when $s(x)$ varies only on long length scales. In such a situation it is appropriate to replace $S=s\left(z w+x_{0}\right)$ in (10)-(13) by $s\left(x_{0}\right)$. For our formulation (10) and (11), after this additional approximation we obtain

$$
\begin{aligned}
\dot{x}_{0} & =\left(\frac{1}{2}-a\right) w s\left(x_{0}\right), \\
\frac{\dot{w}}{3 w}\left(\frac{\pi^{2}}{6}-1\right) & =\frac{1}{24 w^{3}}\left[2-w^{2} s\left(x_{0}\right)\right] .
\end{aligned}
$$

The second of these equations has a stable equilibrium at $w=\sqrt{2 / s\left(x_{0}\right)}$; this in turn implies a value $\dot{x}_{0}=(1-2 a) \sqrt{s / 2}$ for the speed of the front. All these observations agree with properties of the exact solution (5). The Caputo-Sarels ODEs (12) and (13) also reduce correctly in this limit.

\section{B. Wide defects}

To investigate the accuracy of the reduced equations for heterogeneities $s(x)$ that are slowly varying but not small in amplitude, we consider Gaussian and sinusoidal forms

$$
\begin{gathered}
s(x)=s_{0}+s_{1} \exp \left[-x^{2} /(2 d)\right], \\
s(x)=s_{0}+s_{1} \sin (2 \pi x / d) .
\end{gathered}
$$

Figure 1 presents numerical comparisons between the speed and width of the right-traveling front solution, as estimated from a well-converged numerical solution to the PDE (4), and the two approximations (10),(11) and (12),(13). We take the initial condition to be the exact solution (6) centered at $x_{0}=-40$, far from the center of the Gaussian inhomogeneity. We follow the method of [26] by, at a fixed point in time, estimating the best-fit values of $x_{0}$ and $w$ via a least-squares fit of the numerical solution to the kink solution (6). This procedure can be thought of as a projection in the space of available functions. More precisely, we use the MATLAB routine lsqcurvefit that implements an interior-reflective Newton method to solve the nonlinear least-squares problem of fitting the spatial form of the solution $u(x, t)$ at fixed time $t$ to the ansatz (6). This produces the least-squares optimal estimate of the parameters $x_{0}$ and $w$ at that time $t$. The speed of the front $\dot{x}_{0}$ 

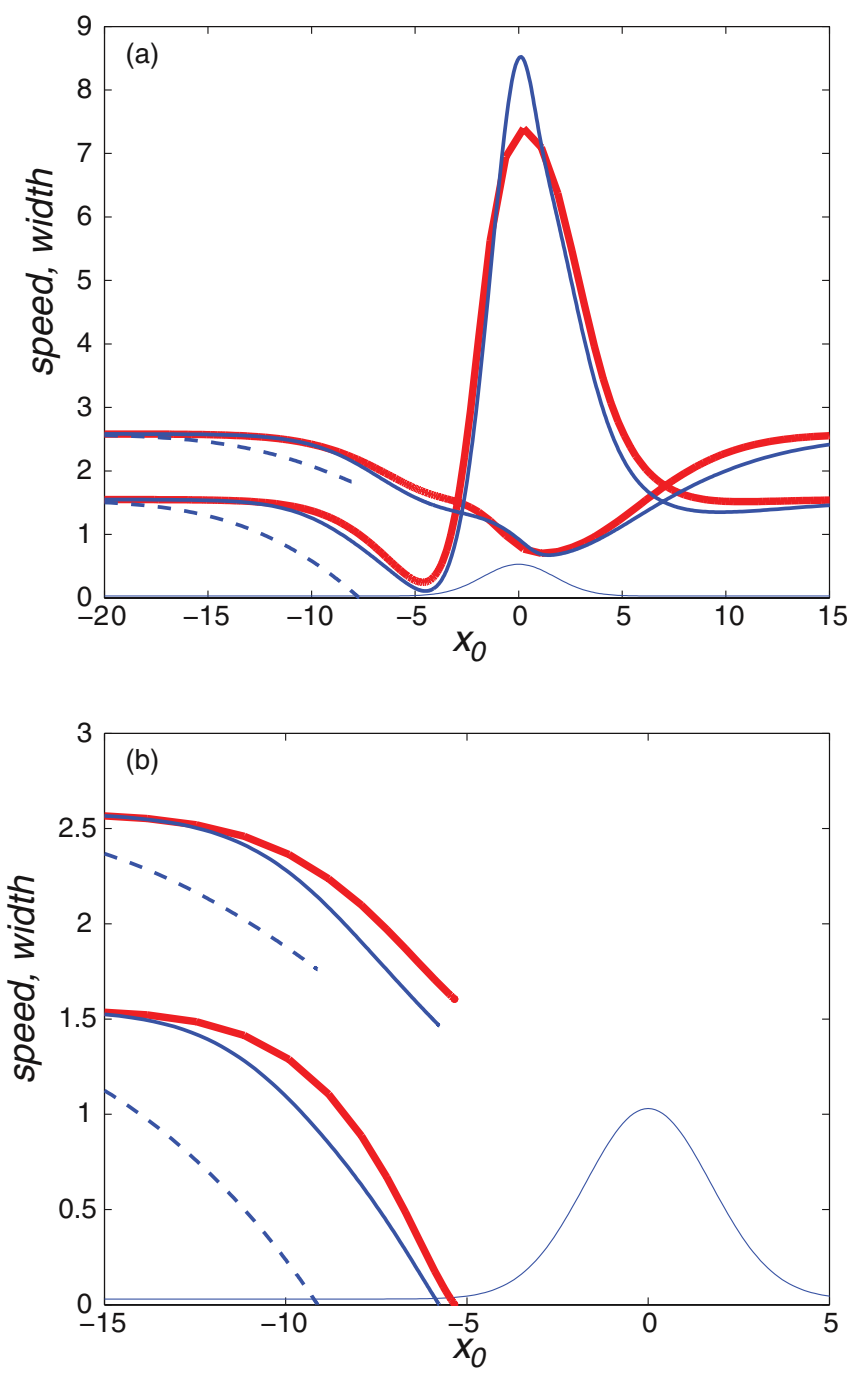

FIG. 1. (Color online) Evolution of the width $w(t)$ and speed $\dot{x}_{0}(t)$ of a front starting at $x_{0}=-40$ and passing through a region of varying $s(x)$, as a function of the position $x_{0}(t)$ of the center of the front, defined to be where $u\left(x_{0}\right)=1 / 2$. Three sets of results are shown: best fit to the numerical solutions (thick solid red curves), the solution of the ODEs (10) and (11) (medium weight solid blue curves), and the solution of the ODEs (12) and (13) (dashed blue curves). In each case the upper line is the width parameter $w(t)$ and the lower is the speed $\dot{x}_{0}(t)$. Also shown is the form of the defect: the plot shows $s(x) / 10$ (thin blue curve). The parameters are $a=s_{0}=0.3, d=3$, and (a) $s_{1}=5$; (b) $s_{1}=10$.

is subsequently estimated by taking a centered second-order finite difference.

Figure 1(a) shows a typical solution in the case of a wide and large-amplitude Gaussian defect, where the front slows rapidly and narrows before accelerating rapidly to move through the region around $x=0$ in which $s(x)$ is larger. The curves all agree closely near $x=-40$ and so for clarity we plot only the range $-15 \leqslant x \leqslant 5$ where their divergence is most obvious. The solution of the reduced system (10) and (11), shown as the solid blue curves, tracks the projection of the numerical solution of the PDE (solid red curves) throughout the motion. In contrast, the solution of the ODEs (12) and (13), shown as
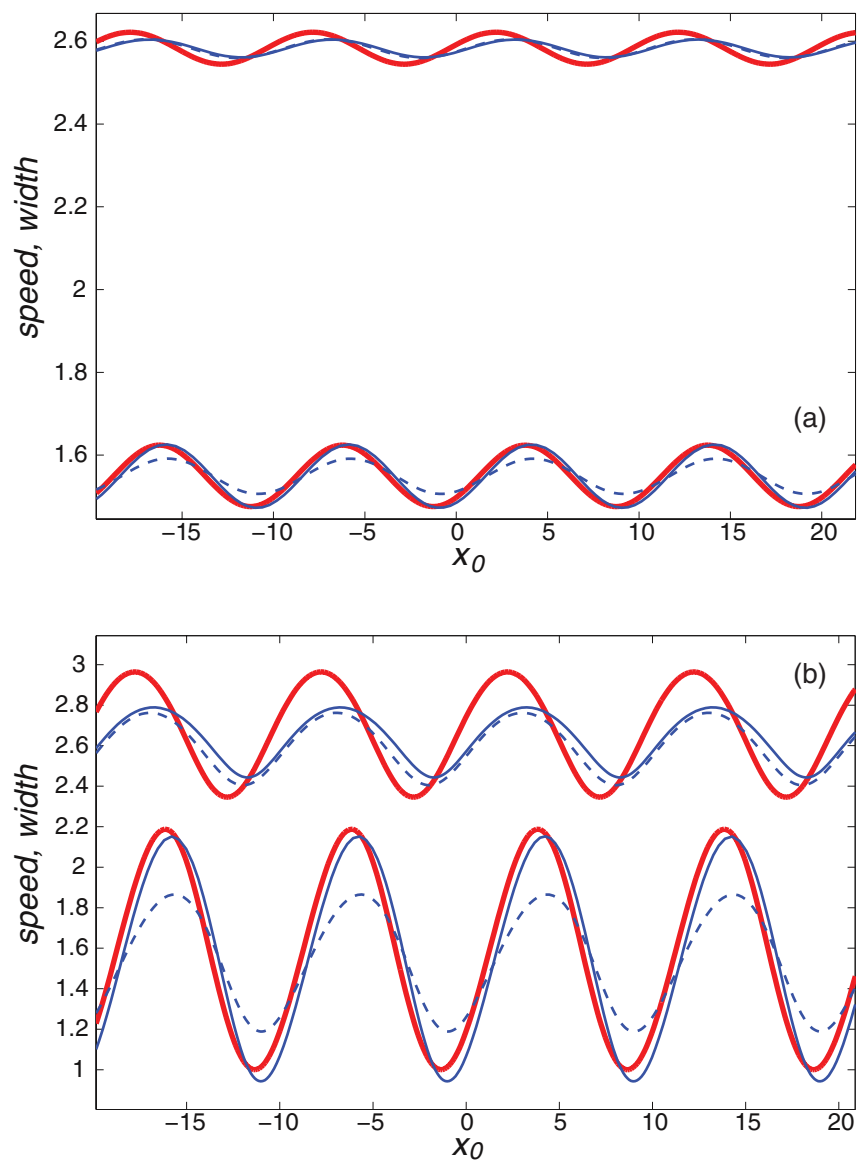

FIG. 2. (Color online) Evolution of the width $w(t)$ and speed $\dot{x}_{0}(t)$ of a front subjected to a sinusoidal variation in $s(x)$, as a function of the position $x_{0}(t)$ of the center of the front, defined to be where $u\left(x_{0}\right)=1 / 2$. The initial condition is (6) centered at $x_{0}=-40$. In each plot we show three sets of results: best fit to the numerical solutions (thick solid red curves), the solution of the ODEs (10) and (11) (medium weight solid blue curves), and the solution of the ODEs (12) and (13) (dashed blue curves). For each line style the upper curve is the width parameter $w(t)$ and the lower is the speed $\dot{x}_{0}(t)$. The parameters are $a=s_{0}=0.3, d=10$, and (a) $s_{1}=0.025$; (b) $s_{1}=0.2$.

dashed blue curves, departs from the (numerical) values much further away from the defect (certainly by the point $x=-10$ ).

Figure 1(b) illustrates the effect of increasing the height of the defect further: the front speed drops to zero, i.e., the front is now pinned near the defect, and the front width remains constant. As in Fig. 1(a) it is clear that the ODEs (10) and (11) provide a much more reliable guide both to the dynamics of the system and to the position at which the front ultimately stops moving, as indicated by the lower curves in the figure falling to zero.

Figure 2 illustrates the accuracy of the approximations when we take the sinusoidal form (15) for the background, instead of the Gaussian. For this numerical simulation we used the initial condition (6) starting at $x_{0}=-40$. In this case the sinusoidal inhomogeneity occupies the entire computational domain $-40 \leqslant x \leqslant 40$ so that in this case the curves depart immediately from each other as the computation begins. Over the range of $x$ shown in Fig. 2 the computations have, however, 
settled into periodic fluctuations that illustrate the nature of the dynamics at long times.

We observe that our variational method produces much better agreement with numerical simulation for the speed of the front (the lower curves) in both plots. Interestingly, the variation in front width is not captured well by either collective coordinate approach, even for small-amplitude variations in $s(x)$ as shown in Fig. 2(a). It appears that the width of the front takes far longer to adjust to variations than does its speed. In contrast, Fig. 2(b) indicates that the variational ODEs (10) and (11) provide a good guide to the evolution of the speed even when the fluctuations in $s(x)$ are very large: note that the fluctuations in $s(x)$ cover the range $0.1 \leqslant s(x) \leqslant 0.5$ in this case.

\section{Narrow Gaussian defects}

As the spatial variation in $s(x)$ becomes more rapid one would expect the traveling front solution to the PDE (4) to depart further from the profile (5).

Figure 3 shows the results of both sets of reduced ODEs in a typical case for a narrower defect. The parameter values used in Fig. 3 correspond to those in Fig. 10 of [26]. Figure 3(a) shows, as in Fig. 1, the width and speed of fronts for the exact numerical solution (solid red curves), the ODEs (12) and (13) (dashed blue curves), and the ODEs (10) and (11) (solid blue curves). It is interesting to observe that neither set of ODEs provides a particularly good guide to the width of the front as it crosses the defect. The variation in the front speed is captured much more closely by (10),(11) than by (12),(13).

Figure 3(b) presents a quantitative comparison for a case with a narrow but much higher Gaussian defect. Although the the solution from the ODEs (10) and (11) (solid blue curves) lies much closer to the true numerically computed one than the approximation from the ODEs (12) and (13) (dashed blue curves), it fails in this case to predict that the front stops; instead it indicates a similar slowing down followed by an abrupt acceleration to that found for the parameters of Fig. 1(a); clearly these parameter values are close to the pinning threshold.

\section{Estimates for front pinning}

In the case of a sufficiently strong and sufficiently spatially localized unimodal inhomogeneity, for example the Gaussian defect (14), we expect that the behavior illustrated in Fig. 1(b) is typical: the front width and position tend to constant values and the front is pinned by the inhomogeneity. Although the integrals in (10) and (11) are not usually available analytically, progress can be made in the special case of a $\delta$ function: $s(x)=\alpha+\beta \delta(x)$. For this pointlike inhomogeneity we pose the following question: how large must $\beta$ be in order to pin the front, and what is the resulting position of the front, when pinned? Substituting the pointlike ansatz into (10) and (11), we obtain

$$
\frac{\dot{x}_{0}}{6 w}=\frac{\beta}{w} \frac{e^{-2 x_{0} / w}\left(1-a-a e^{-x_{0} / w}\right)}{\left(1+e^{-x_{0} / w}\right)^{5}}+\frac{\alpha}{12}(1-2 a),
$$
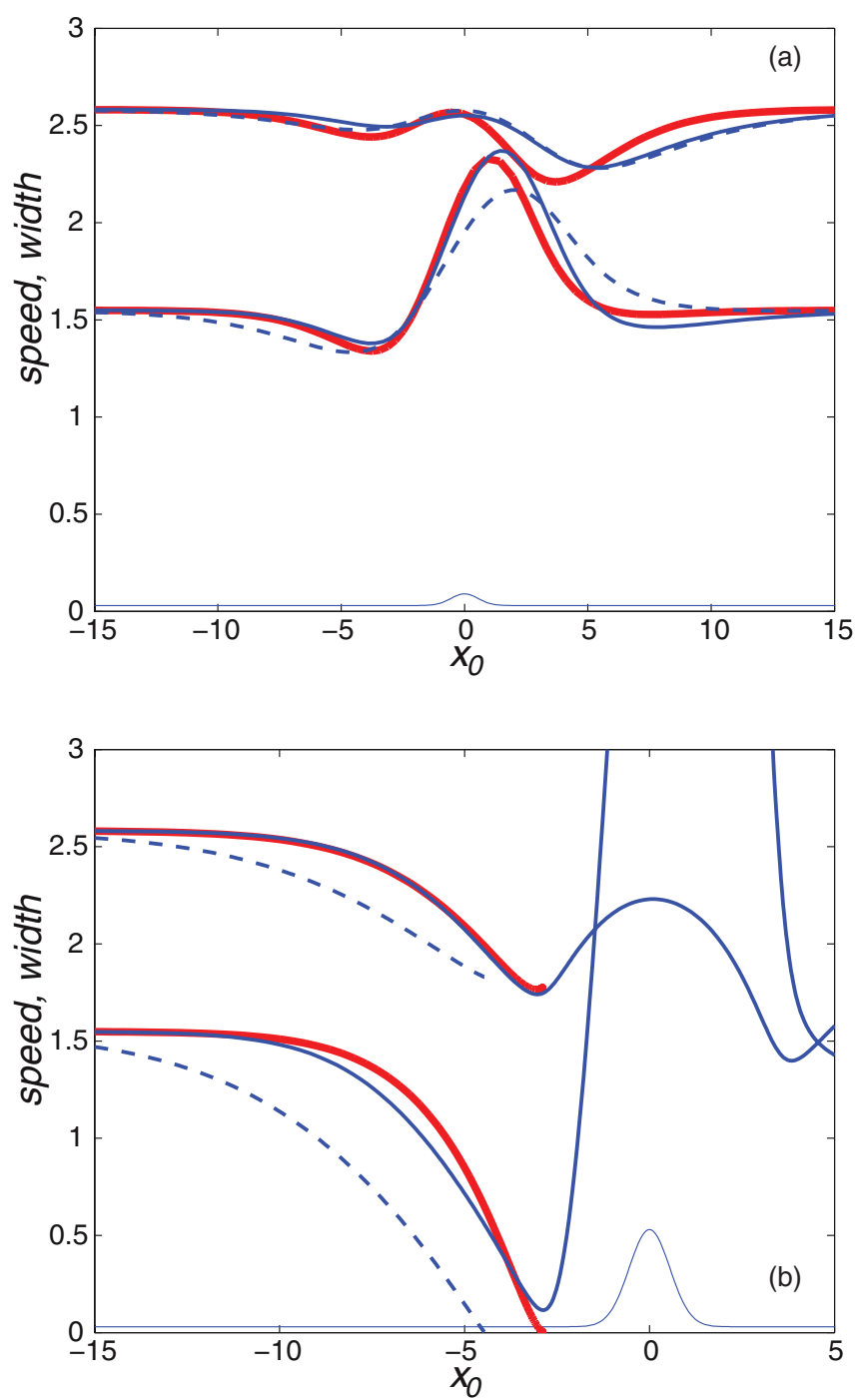

FIG. 3. (Color online) Evolution of the width $w(t)$ and speed $\dot{x}_{0}(t)$ of a front, with initial condition (6), starting at $x_{0}=-40$, and passing through a region of varying $s(x)$, as for Fig. 1 . Note that the speed $\dot{x}_{0}$ is shown increased by a factor of 10 for clarity, and that the defect is shown reduced by a factor of $10: 10 \dot{x}_{0}$ and $s(x) / 10$ are plotted. Line colors and styles are as in Fig. 1. The parameters are $a=s_{0}=d=0.3$ and (a) $s_{1}=0.6$; (b) $s_{1}=5$.

$$
\begin{aligned}
\frac{\dot{w}}{3 w}\left(\frac{\pi^{2}}{6}-1\right)= & \frac{1}{12 w^{3}}-\frac{\alpha}{24 w} \\
& -\frac{\beta x_{0}}{w^{3}} \frac{e^{-2 x_{0} / w}\left(1-a-a e^{-x_{0} / w}\right)}{\left(1+e^{-x_{0} / w}\right)^{5}} .
\end{aligned}
$$

For a pinned front we require $\dot{x}_{0}=\dot{w}=0$. Looking for equilibria of (16) and (17) we write (16) in the form

$$
g\left(x_{0} / w\right)=-\frac{\alpha w}{12 \beta}(1-2 a),
$$

where we define

$$
g\left(x_{0} / w\right):=\frac{e^{-2 x_{0} / w}\left(1-a-a e^{-x_{0} / w}\right)}{\left(1+e^{-x_{0} / w}\right)^{5}} .
$$

We may now estimate the critical (i.e., smallest positive) value of $\beta$ required to pin the front by minimizing $g\left(x_{0} / w\right)$ over its 
argument. The relevant turning point of the two available is the one at the more negative value of $z \equiv x_{0} / w$. We obtain $z=$ $z_{\min }=\ln \{(3-r) /[4(1-a)]\}$ where $r=\sqrt{16 a(a-1)+9}$. Hence

$$
g\left(z_{\min }\right)=\frac{16(3-r-4 a)(3-r)^{2}(1-a)^{3}}{(7-r-4 a)^{5}} .
$$

Substituting $x_{0} / w=z_{\min }, g=g\left(z_{\min }\right)$, and $\beta=\beta_{c}$ into (17) yields

$$
\beta_{c} z_{\min } g\left(z_{\min }\right)=\frac{1}{12 w}-\frac{\alpha w}{24},
$$

which can be solved for $w$ to give

$$
w_{\min }=\left(\frac{2 / \alpha}{1-2(1-2 a) z_{\min }}\right)^{1 / 2} .
$$

We can now write down the corresponding estimate of the pinning position $x_{0}$ since $x_{0}^{\min }=w_{\min } z_{\min }$. Moreover, the critical value $\beta_{c}$ can be estimated using the relation (18) which was not directly used in the last few lines above. We obtain the explicit estimates

$$
\begin{aligned}
\beta_{c}= & \left(\frac{\alpha / 2}{1-2(1-2 a) z_{\min }}\right)^{1 / 2} \\
& \times \frac{(1-2 a)(7-r-4 a)^{5}}{96(4 a+r-3)(3-r)^{2}(1-a)^{3}}, \\
x_{0}^{\min } & =\left(\frac{2 / \alpha}{1-2(1-2 a) z_{\min }}\right)^{1 / 2} z_{\min },
\end{aligned}
$$

where $\quad z_{\min } \equiv \ln \{(3-r) /[4(1-a)]\} \quad$ and $\quad r=$ $\sqrt{16 a(a-1)+9}$ depend only on the PDE parameter $a$. Similar estimates were obtained by Caputo and Sarels [26] for their ODE system; see their Eqs. (31) and (32).

Figure 4 presents comparisons of the numerically determined location $x_{0}^{\text {min vel }}$ at which a front attains its minimum velocity while moving over the defect. For large enough $s_{1}$, above the value at which $x_{0}^{\min }$ vel achieves its maximum, the fronts are pinned and come to rest at the locations indicated. The results of the ODEs (10) and (11) and the estimates provided by (20) are compared with numerical values. The figure also indicates, with the vertical lines, the estimates of the minimum value of $s_{1}$ required in order to achieve pinning. The locations of the vertical lines should be compared to the locations of the maxima in the curves traced out by the (red) squares, (black) circles, and (blue) asterisks. Note that the black vertical dashed line obtained from the analytical estimation using the pointlike $\delta$-function ansatz is close to the maxima in the red and black curves in the upper part of Fig. 4, for which the defect is more sharply localized than in the lower part of the figure.

More precisely, in order to compare the results for a Gaussian (14) with the $\delta$-function form, we equate the integrals of $s(x)$ in the two cases. This implies the correspondence $\beta=s_{1} \sqrt{2 \pi d}$. In both parts of Fig. 4 we observe that the variational ODEs are a substantially better guide to the pinning location than that given in [26], although both give qualitatively a correct description of the behavior. The $\delta$-function approximation is, as one might expect, substantially closer in Fig. 4(a) for which $d=0.1$ and so the inhomogeneity occurs over a length scale smaller than the front width and much more like a $\delta$ function. For larger $d$ [Fig. 4(b)], the
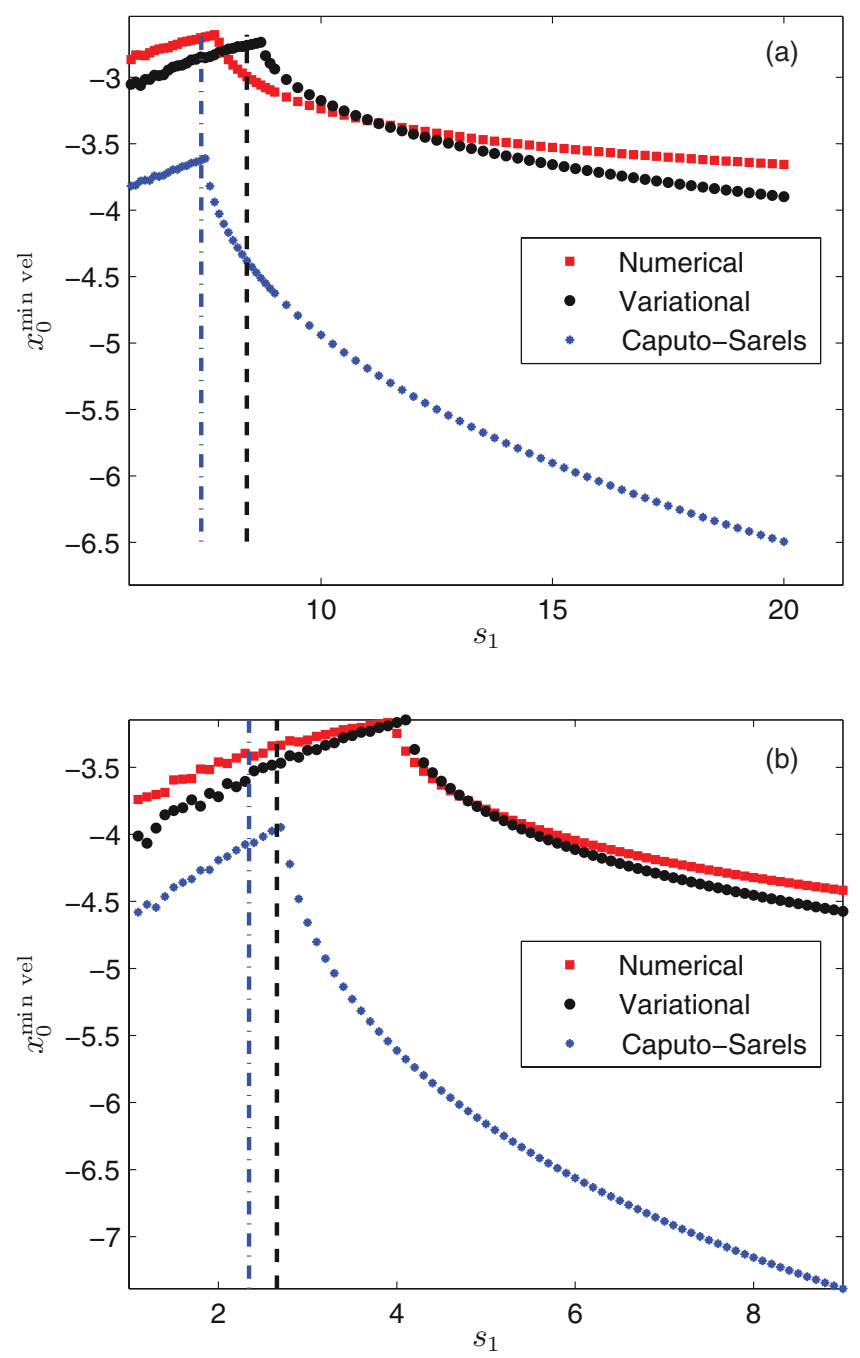

FIG. 4. (Color online) Location of the minimum value of $\dot{x}_{0}$ as a function of $s_{1}$ for the Gaussian inhomogeneity (14). Where the data values are increasing with increasing $s_{1}$ the front is not pinned, but attains a minimum velocity at the point shown. After the maximum data value, in the range where the data are decreasing as $s_{1}$ increases, the front is pinned (i.e., attains zero velocity) at the point shown. Data are shown for numerical simulations (squares, red), the ODEs (10) and (11) (circles, black), and the ODEs (12) and (13) derived by [26] (asterisks, blue). The vertical lines indicate the estimates of the critical (i.e., lowest) $s_{1}=\beta_{c} / \sqrt{2 \pi d}$ at which the front is pinned. The variational (20) and Caputo-Sarels estimates are shown as the black dashed and blue dot-dashed lines, respectively. The parameters are $a=s_{0}=0.3$ and (a) $d=0.1$; (b) $d=1$.

value of $s_{1}$ needed to pin the front decreases substantially. The values of $x_{0}$ at which the front pins do not change significantly between Figs. 4(a) and 4(b); this agrees with the expression (21) for $x_{0}^{\min }$ which depends only on the parameters $a$ from the PDE and $\alpha$ which is the limiting "far-field" value of $s(x)$.

\section{CONCLUSION}

In this paper we have discussed the relation between the variational approximation and collective coordinate methods. We showed that the variational method implies a natural choice of functions to use in deriving a reduced description of the 
dynamics in terms of collective coordinates. Our method also extends in a natural way to nonvariational problems; in this work this extension is not used, but it points the way to a unified approach for both variational and nonvariational situations that we believe is a scientifically valuable observation. For the specific problem considered in Sec. III, this formulation of the collective coordinate reduction yielded a much more accurate approximation than an existing treatment in the literature [26].

Many questions arise which we will return to in future work. First, we have not provided any systematic approach to estimating how accurate we might expect this approach to be. Bounds on the accuracy that one might expect would be extremely useful. Error bounds for variational approximations for Lagrangian systems having equilibrium or traveling wave solutions have been given in $[37,38]$. To our knowledge, the only existing analyses for the fully dynamic case similar to that here only consider slowly varying position of the front, i.e., $x_{0}(t)$, (see, e.g., [39-42]). Second, it is questionable whether or not the method that we outline above maintains its accuracy when there are different length scales present in the problem, since previous work [25] has indicated that competition between different length scales may strongly influence the applicability of collective coordinate methods [25]. One specific example of this problem is the subcritical (and non-variational) Swift-Hohenberg equation, which is well known to admit spatially localized equilibrium, traveling, and time-periodically pulsating states that have spatially oscillating tails $[43,44]$. Inspired by the results of multiple-scale asymptotics, variational formulations of the problem have enabled the development of good approximations to the dynamics of the localised states $[45,46]$. One natural extension of this work would be to analyze the interaction of such localized states with background inhomogeneities of the kind discussed in this paper.

\section{ACKNOWLEDGMENTS}

J.H.P.D. acknowledges support from the Royal Society through a University Research Fellowship.
[1] C. S. Gardner, J. M. Greene, M. D. Kruskal, and R. M. Miura, Phys. Rev. Lett. 19, 1095 (1967).

[2] J. Boussinesq, Acad. Sci. Inst. Nat. France 23, 1 (1877).

[3] D. J. Korteweg and G. de Vries, Philos. Mag. 39, 422 (1895).

[4] M. J. Ablowitz, D. J. Kaup, A. C. Newell, and H. Segur, Phys. Rev. Lett. 30, 1262 (1973).

[5] M. J. Ablowitz, D. J. Kaup, A. C. Newell, and H. Segur, Stud. Appl. Math. 53, 249 (1974).

[6] V. E. Zakharov and A. B. Shabat, Sov. Phys. JETP 34, 62 (1972),

[7] V. E. Zakharov and A. B. Shabat, Sov. Phys. JETP 37, 823 (1973).

[8] J. P. Wang, J. Nonlin. Math. Phys. 9, 213 (2002).

[9] N. J. Zabusky and M. D. Kruskal, Phys. Rev. Lett. 15, 240 (1965).

[10] F. Calogero and A. Degasperis, Lett. Nuovo Cimento Soc. Ital. Fis. 16, 425 (1976).

[11] H.-H. Chen and C.-S. Liu, Phys. Rev. Lett. 37, 693 (1976).

[12] V. N. Serkin, A. Hasegawa, and T. L. Belyaeva, Phys. Rev. Lett. 98, 074102 (2007).

[13] V. I. Karpman, Pis'ma Zh. Eksp. Teor. Phys. 25, 296 (1977) [Sov. Phys. JETP Lett. 25, 271 (1977)].

[14] V. I. Karpman and E. M. Maslov, Zh. Eksp. Teor. Fiz. 73, 537 (1977) [Sov. Phys. JETP 46, 281 (1977)].

[15] D. J. Kaup, SIAM J. Appl. Math. 31, 121 (1976).

[16] Yu. S. Kivshar and B. A. Malomed, Rev. Mod. Phys. 61, 763 (1989).

[17] D. J. Bergman, E. Ben-Jacob, Y. Imry, and K. Maki, Phys. Rev. A 27, 3345 (1983).

[18] D. W. McLaughlin and A. C. Scott, Phys. Rev. A 18, 1652 (1978).

[19] N. R. Pereira and L. Stenflo, Phys. Fluids 20, 1733 (1977).

[20] C. Chong and D. E. Pelinovsky, Discr. Contin. Dyn. Syst. Series S 4, 1019 (2011).

[21] B. A. Malomed, Prog. Opt. 43, 71 (2002).

[22] M. J. Rice and E. J. Mele, Solid State Commun. 35, 487 (1980).

[23] M. J. Rice, Phys. Rev. B 28, 3587 (1983).

[24] S. Chandrasekhar, Hydrodynamic and Hydromagnetic Stability (Oxford University Press, Oxford, 1961, republished by Dover, New York, 1981).
[25] A. Sánchez and A. R. Bishop, SIAM Rev. 40, 579 (1998).

[26] J.-G. Caputo and B. Sarels, Phys. Rev. E 84, 041108 (2011).

[27] R. Boesch, P. Stancioff, and C. R. Willis, Phys. Rev. B 38, 6713 (1988).

[28] R. Boesch, C. R. Willis, and M. El-Batanouny, Phys. Rev. B 40, 2284 (1989).

[29] E. Ott and R. N. Sudan, Phys. Fluids 12, 2388 (1969).

[30] D. Anderson, A. Bondeson, and M. Lisak, Phys. Lett. A 67, 331 (1978).

[31] A. Bondeson, M. Lisak, and D. Anderson, Phys. Scr. 20, 479 (1979).

[32] S. Chavez Cerda, S. B. Cavalcanti, and J. M. Hickmann, Eur. J. Phys. D 1, 313 (1998).

[33] Yu. S. Kivshar and W. Krolikowski, Opt. Commun. 114, 353 (1995).

[34] A. K. Abramian and S. A. Vakulenko, Theor. Math. Phys. 155, 678 (2008).

[35] S. Vakulenko, Manu, J. Reinitz, and O. Radulescu, Phys. Rev. Lett. 103, 168102 (2009).

[36] G. B. Whitham, Linear and Nonlinear Waves (WileyInterscience, New York, 1974).

[37] C. Chong, D. Pelinovsky, and G. Schneider, Physica D 241, 115 (2012).

[38] D. J. Kaup and T. K. Vogel, Phys. Lett. A 362, 289 (2007).

[39] S.-I. Ei, J. Dyn. Differ. Eq. 14, 85 (2002).

[40] S.-I. Ei, M. Mimura, and M. Nagayama, Physica D 165, 176 (2002).

[41] S. A. Vakulenko, J. Sov. Math. 57, 3093 (1991).

[42] S. A. Vakulenko and P. V. Gordon, Theor. Math. Phys. 112, 1104 (1997).

[43] J. Burke and J. H. P. Dawes, SIAM J. Appl. Dyn. Syst. 11, 261 (2012).

[44] J. H. P. Dawes, Philos. Trans. R. Soc., A 368, 3519 (2010)

[45] P. C. Matthews and H. Susanto, Phys. Rev. E 84, 066207 (2011).

[46] H. Susanto and P. C. Matthews, Phys. Rev. E 83, 035201(R) (2011). 